\title{
Full Duplex Techniques for 5G Networks: Self-Interference Cancellation, Protocol Design, and Relay Selection
}

\author{
Zhongshan Zhang, Xiaomeng Chai, Keping Long, Athanasios V. Vasilakos, and Lajos Hanzo
}

Zhongshan Zhang,

Xiaomeng Chai, and Keping Long are with the University of Science and Technology Beijing.

Athanasios V. Vasilakos is with the University of Western Macedonia.

Lajos Hanzo is with the University of Southampton.

This work was supported by the key project of the National Natural Science Foundation of China (No. 61431001), the 863 project No.

2014AA01A701, Program for New Century

Excellent Talents in University (NECT-12-0774),

the open research fund of National Mobile

Communications Research Laboratory Southeast University (No. 2013D12), Fundamental Research Funds for the Central Universities, and the Foundation of Beijing Engineering and Technology Research Center for Convergence Networks.

\begin{abstract}
The wireless research community aspires to conceive full duplex operation by supporting concurrent transmission and reception in a single time/frequency channel for the sake of improving the attainable spectral efficiency by a factor of two as compared to the family of conventional half duplex wireless systems. The main challenge encountered in implementing FD wireless devices is that of finding techniques for mitigating the performance degradation imposed by self-interference. In this article, we investigate the potential FD techniques, including passive suppression, active analog cancellation, and active digital cancellation, and highlight their pros and cons. Furthermore, the troubles of FD medium access control protocol design are discussed for addressing the problems such as the resultant end-to-end delay and network congestion. Additionally, an opportunistic decode-andforward-based relay selection scheme is analyzed in underlay cognitive networks communicating over independent and identically distributed Rayleigh and Nakagami- $m$ fading channels in the context of FD relaying. We demonstrate that the outage probability of multi-relay cooperative communication links can be substantially reduced. Finally, we discuss the challenges imposed by the aforementioned techniques and a range of critical issues associated with practical FD implementations. It is shown that numerous open challenges, such as efficient SI suppression, high-performance FD MAC-layer protocol design, low power consumption, and hybrid FD/HD designs, have to be tackled before successfully implementing FD-based systems.
\end{abstract}

\section{INTRODUCTION}

The spectral efficiency (SE) of networks has to be further improved in order to deliver ever increasing data rates. However, the operational wireless communication systems usually rely on half duplex (HD) operations, leading to erosion of resource exploitation. The promise of radical full duplex (FD) operation, on the other hand, improves the achievable SE of wireless communication systems by always transmitting and receiving in the entire bandwidth.

The main driving force behind the advances in FD communications is the promise of nearly doubled channel capacity compared to conventional HD communications, thus offering the potential to complement and sustain the evolution of the fifth generation $(5 \mathrm{G})$ technologies toward denser heterogeneous networks with flexible relaying modes [1]. Recently, a range of theoretical and practical aspects of FD communications have been investigated by quantifying the performance gains of FD modes (FDMs) [2], which exhibits advantages over the half-duplex mode (HDM) in terms of either having increased throughput or reduced outage probability (OP), albeit achieved at the cost of increased complexity. Furthermore, recent advances in FD communications have increased both the attainable throughput and the diversity orders of wireless communication systems. Once increased hardware/software complexity is tolerated to facilitate more sophisticated signal processing, it would be possible for an FD device to reduce the bit error rate (BER). In addition, the packet loss ratio (PLR) of FDM may also be reduced, provided that a larger buffer size is provided by FD devices.

However, as a downside, the FD gain is eroded by self-interference (SI) due to the large power difference between the power imposed by a device's own transmissions and the low-power received signal arriving from a remote transmit antenna. Excessive SI may even result in reduced capacity for FD systems that falls below that of HD systems. Consensus reached by both industry and academia show that it is critical to perform efficient SI suppression/cancellation in implementing radical FD communication systems. Apart from the aforementioned physicallayer issues, the conception of FD medium access control (MAC) protocols requires substantial further research. Experience indicates that FD schemes may not always outperform their HD counterparts, and hybrid schemes that switch between HDM and FDM can also be 

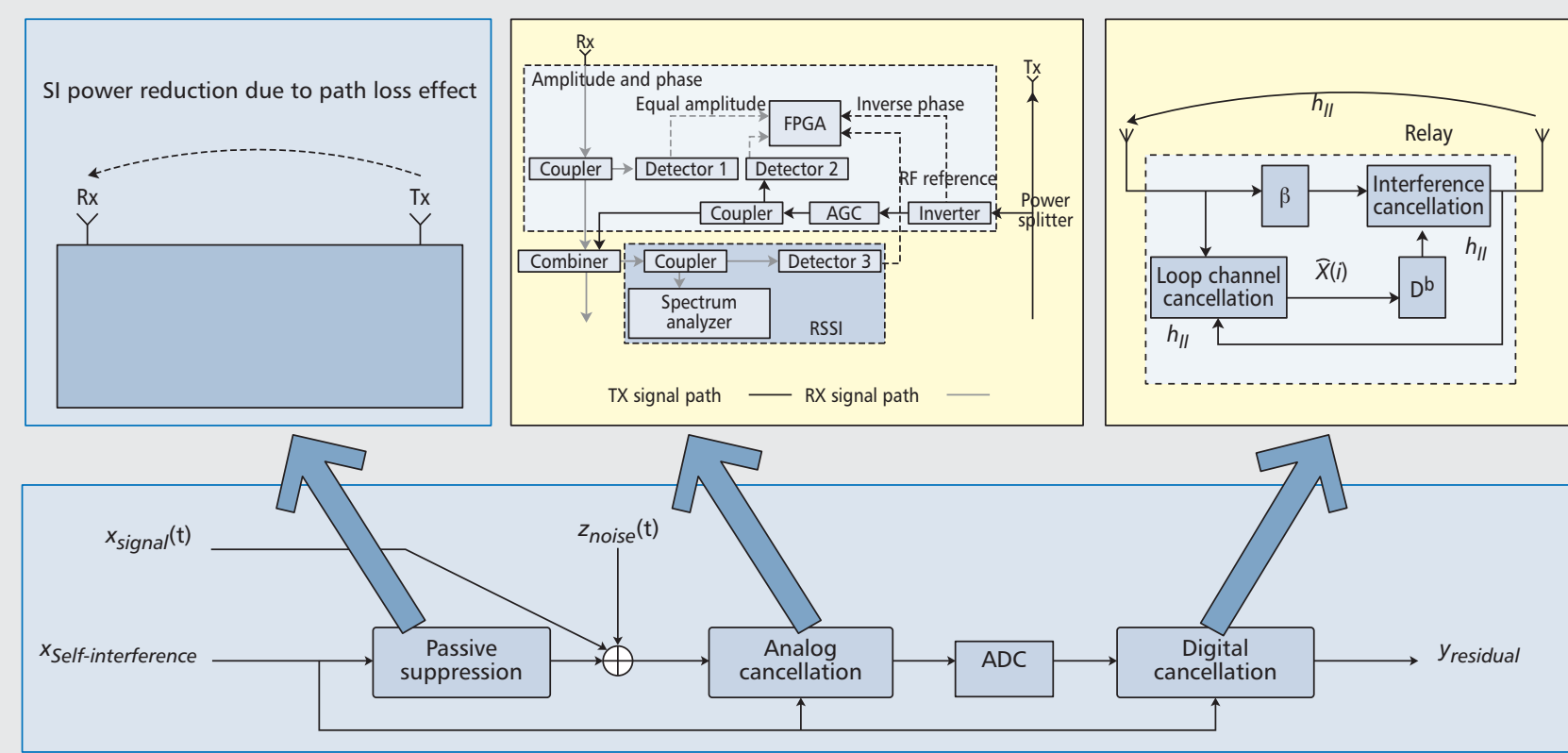

\begin{tabular}{|c|c|c|c|c|c|c|c|}
\hline Algorithm & \begin{tabular}{|l} 
Transmit \\
power
\end{tabular} & $\begin{array}{l}\text { Center } \\
\text { frequency }\end{array}$ & Bandwidth & $\begin{array}{l}\text { Antenna } \\
\text { distances }\end{array}$ & $\begin{array}{l}\text { Antenna } \\
\text { separations }\end{array}$ & $\begin{array}{l}\text { Cancellation } \\
\text { capability }\end{array}$ & $\begin{array}{l}\text { Full duplex } \\
\text { gain }\end{array}$ \\
\hline \multirow{2}{*}{$\begin{array}{l}\text { Compact/separated } \\
\text { antennas }\end{array}$} & \multirow{2}{*}{$5 \mathrm{dBm}$} & \multirow[t]{2}{*}{$2.6 \mathrm{GHz}$} & \multirow{2}{*}{$100 \mathrm{MHz}$} & $20 \mathrm{~mm}$ & & $48 \mathrm{~dB}$ & \\
\hline & & & & $5 \mathrm{~m}$ & & $70 \mathrm{~dB}$ & \\
\hline \multirow{2}{*}{$\begin{array}{l}\text { Antenna } \\
\text { cancellation }\end{array}$} & & \multirow[t]{2}{*}{$2.4 \mathrm{GHz}$} & \multirow[t]{2}{*}{$100 \mathrm{MHz}$} & $\lambda / 2$ & & $60 \mathrm{~dB}$ & 1.84 \\
\hline & & & & & & & \\
\hline ZigZag & & $2.4 \mathrm{GHz}$ & & & & & 1.25 \\
\hline \multirow{2}{*}{$\begin{array}{l}\text { Antenna } \\
\text { separation }\end{array}$} & \multirow{2}{*}{$\begin{array}{l}-5 \mathrm{dBm} \\
\sim 15 \mathrm{dBm}\end{array}$} & \multirow[t]{2}{*}{$2.4 \mathrm{GHz}$} & \multirow[t]{2}{*}{$625 \mathrm{kHz}$} & $20 \mathrm{~cm}$ & & $39 \mathrm{~dB}$ & \\
\hline & & & & $40 \mathrm{~cm}$ & & $45 \mathrm{~dB}$ & \\
\hline \multirow{2}{*}{$\begin{array}{l}\text { Directional } \\
\text { diversity }\end{array}$} & \multirow[t]{2}{*}{$12 \mathrm{dBm}$} & \multirow[t]{2}{*}{$2.4 \mathrm{GHz}$} & \multirow[t]{2}{*}{$20 \mathrm{MHz}$} & $10 \mathrm{~m}$ & $\geq 45^{\circ}$ & & $1.6 \sim 1.9$ \\
\hline & & & & $15 \mathrm{~m}$ & $\geq 90^{\circ}$ & & $\geq 1.4$ \\
\hline
\end{tabular}

Figure 1. Practical implementable SI suppression algorithms and their performance comparison.

developed for adaptively exploiting the radio resources, while at the same time maximizing the SE [3]. Again, an FD scheme may not always outperform its HD counterpart, requiring a hybrid HD/FD scheme to be implemented to gain an advantage over either of the individual schemes. In this article, we survey/compare different FD techniques. Some existing SI cancellation techniques such as passive suppression, active analog, and digital cancellation are discussed. Furthermore, the critical issues associated with FD-based MAC-layer protocols are also studied. Finally, the choice of the optimal relay selection scheme conceived for FDM is elaborated on, followed by a variety of new directions and open problems. The main contributions of this article include

- Surveying the critical issues related to FD transmissions from a physical-layer perspective relying on SI suppression

- Giving cognizance to the MAC-layer protocols

- Proposing an FD-based opportunistic decode-and-forward (DF)-based relay selection scheme in the context of underlay cognitive networks and analyzing the $\mathrm{OP}$ of the multi-relay cooperative communication links

- Outlining several challenges associated with
FDM-based device/system realizations

- Discussing both the advantages and drawbacks of various FD techniques, while identifying their challenges and new directions The remainder of this article is organized as follows. The classification of both passive and active SI suppression is detailed next. Typical FD MAC-layer protocols, such as the FD-MAC technique [4], are then discussed, followed by several critical issues related to the associated practical implementation and commercial realizations. We then propose an opportunistic FDM relay selection scheme, followed by a range of open challenges and the future directions of FD communications. Finally, our conclusions are provided.

\section{Self-InTERference CanCELLation}

Existing studies $[5,6]$ showed that it is critical to accurately measure and suppress the SI in FD communication. For instance, as revealed in [7], the SI power as well as spatial reuse may substantially reduce the FD gain over the HDM in terms of the network-level capacity, rendering it well below 2 in common cases. However, if the SI level at the input of the FD relay (i.e., after performing SI suppression) can be at least $3 \mathrm{~dB}$ lower than the noise level, the remaining SI may 
not seriously degrade the end-to-end throughput [8]. SI cancellation techniques are usually classiSince the analog SI cancellation methods are never perfect, the residual SI after analog cancellation should be further reduced with the aid of digital cancellation. In the existing digital-cancellation protocols, ZigZag exhibits a significant advantage in terms of the achievable FD gains.

fied into passive and active suppressions, as shown in Fig. 1.

\section{PASSIVE SI SUPPRESSION}

Passive SI suppression is defined as the signalpower attenuation imposed by the path loss due to the physical separation between the transmit and receive antennas of the same device. Typical passive SI suppression techniques include:

Directional SI suppression: In this technique, the main radiation lobes of the transmit/receive antennas of an FD device have minimal intersection, enabling the SI to be partially suppressed prior to the receiver's RF front-end.

Antenna separation and SI cancellation: Increasing the path loss between the transmit/ receive antennas constitutes an effective approach to attenuate the SI power, in which method a higher antenna separation implies better SI suppression performance. When relying on antenna separation, the natural isolation may also exploit the surrounding buildings or the beneficial inclusion of a shielding plate, provided that strict restrictions imposed on the device size can be satisfied.

\section{Active Self-InTERference SUPPRESSION}

In [9], active SI suppression methods were shown experimentally to be capable of facilitating FD communication at ranges up to $6 \mathrm{~m}$ and at transmit powers typical of WiFi devices, revealing that the interference level can be reduced by 50 $\mathrm{dB}$ and $40 \mathrm{~dB}$ under static and dynamically fading interference channel scenarios, respectively, if an RF SI canceller is combined with a baseband canceller. The family of active suppression techniques can be subdivided into analog cancellation, digital cancellation, and combined ana$\log$ /digital cancellation, as discussed below.

Analog Cancellation - In analog cancellation, the family of time-domain (TD) cancellation algorithms such as training-based methods can be employed by both single-input single-output (SISO) and multiple-input multiple-output (MIMO) based techniques, where the latter may perform SI suppression by exploiting the spatial diversity achieved by the associated multiple transmit and/or receive antennas.

- Classic TD training-based methods can be beneficially utilized for estimating the SI leakage, while facilitating reliable SI cancellation. Asymmetric complex signals, in which the inputs are chosen to be complex but not circularly symmetric, can also be utilized for mitigating the SI in single-antenna-aided FDM relays under DF relaying. The optimum SI cancellation weight vectors can be exploited by increasing the signalto-noise ratio (SNR) of the source $\rightarrow$ relay and relay $\rightarrow$ destination links, thus beneficially improving the attainable throughput of FD relaying channels.

- The increased degree of freedom (DoF) offered by the spatial domain (SD) antenna arrays of MIMO systems may be utilized to provide a range of new solutions for SI cancellation. In MIMO aided FD systems, relays are capable of operating in either the antenna-partitioning- based mode (i.e., all antennas operating in the FDM but partitioned into transmit and receive antenna sets) or antenna-sharing-based mode (i.e., allowing antennas to be utilized more efficiently by exploiting the increased dimensions of MIMO channels and/or by relying on time-division duplexing, TDD, aided channel reciprocity).

Digital Cancellation - Since analog SI cancellation methods are never perfect, the residual SI after analog cancellation should be further reduced with the aid of digital cancellation. Of the existing digital cancellation protocols, ZigZag [10] exhibits a significant advantage in terms of the achievable FD gains. Note that ZigZag imposes no change on the conventional IEEE 802.11 MAC protocols when there is no collision, thus maintaining the same throughput as if the colliding packets were scheduled a priori in separate time slots in the presence of transmission collisions. It has been observed that 10 percent of the transmitter-receiver pairs of a wireless network often experience severe packet loss due to packet collisions imposed by statistical channel multiplexing. The asynchronous nature of successive collisions can be successfully exploited in ZigZag to address the problem of high packet loss rate (PLR). By using ZigZag, the average PLR at hidden terminals was shown to be reduced from 72.6 to about 0.7 percent, while improving the average throughput by 25.2 percent compared to the conventional IEEE 802.11 standards.

Performance Comparison - The SI suppression capabilities of some typical algorithms are characterized in Fig. 1. Although numerous sophisticated techniques have been proposed for performing SI cancellation in FD devices, both advantages and disadvantages are exhibited in the context of each approach, as shown in Table 1.

OPEN ReSEARCh ISSUES IN SI SUPPRESSION

Although passive SI suppression techniques are capable of attenuating the SI power in proportion to the path loss, enabling a higher antenna separation usually requires a larger or even infeasible device size. More detrimentally, increasing the antenna separation implies a degradation of the SI channel estimation. Furthermore, numerous additional challenges have to be addressed in the context of the existing active SI suppression techniques. For instance, the achievable SI cancellation capability may be limited by relying on standalone analog or digital cancellation. It is thus rather critical to effectively balance the roles of the analog- and digitaldomain functions in the overall SI cancellation, carefully revealing the overall benefits of combined analog/digital cancellation. In the following, a number of possible solutions to the above-mentioned challenges should be proposed.

Antenna configuration for practical size-limited FD devices: In passive SI suppression schemes, the best antenna configuration in terms of the attainable SI suppression can be achieved upon installing the transmit and receive antennas at the opposite sides of the device to create sufficient separation, requiring the device size to be large enough. 


\begin{tabular}{|c|c|c|c|c|c|}
\hline \multicolumn{2}{|c|}{ Category } & Algorithm & $\mathbf{T}_{\mathbf{x}} \times \mathbf{R}_{\mathbf{x}}$ & Advantage & Disadvantage \\
\hline \multicolumn{2}{|c|}{ 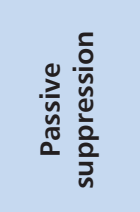 } & $\begin{array}{l}\text { Directional } \\
\text { diversity } \\
\text { Antenna } \\
\text { separation }\end{array}$ & & $\begin{array}{l}\text { 1) SI attenuated due to path loss } \\
\text { 2) Decreases inter-device interference } \\
\text { 3) Improves power efficiency } \\
\text { 4) More separation implies a better attenuation } \\
\text { of SI signal }\end{array}$ & $\begin{array}{l}\text { 1) Performance depends highly on AS and } \\
\text { beam pattern } \\
\text { 2) AS is restricted by variant factors such } \\
\text { as device size and interference channel } \\
\text { estimation accuracy } \\
\text { 3) Restriction applications to SISO }\end{array}$ \\
\hline \multirow{11}{*}{ 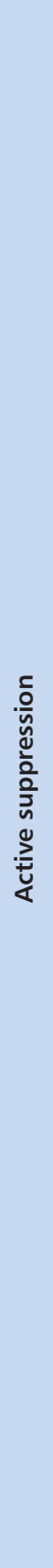 } & \multirow{10}{*}{ 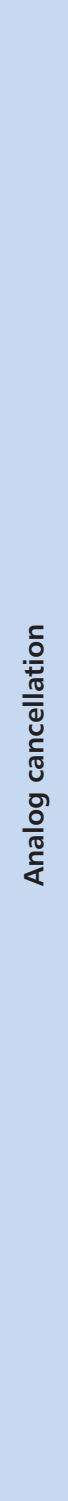 } & $\begin{array}{l}\text { Antenna } \\
\text { Cancellation }\end{array}$ & $2 \times 1$ & $\begin{array}{l}\text { 1) Easy to implement } \\
\text { 2) High cancellation capability } \\
\text { 3) Robust in narrowband systems }\end{array}$ & $\begin{array}{l}\text { 1) Broadband-induced loss } \\
\text { 2) Degrades the received signal } \\
\text { 3) Limited transmit power } \\
\text { 4) Requires fixed AS }\end{array}$ \\
\hline & & Pre-Nulling & $M \times 1$ & $\begin{array}{l}\text { 1) Simple to implement } \\
\text { 2) No influence on receiver BER } \\
\text { 3) Stringent requirements on antenna isolation } \\
\text { are required }\end{array}$ & $\begin{array}{l}\text { 1) SI channel estimation is required } \\
\text { 2) Designed specifically for flat-fading } \\
\text { channels }\end{array}$ \\
\hline & & AFC & $1 \times M$ & $\begin{array}{l}\text { 1) Low complexity } \\
\text { 2) Needs no training sequence } \\
\text { 3) No delay insertion in the relay } \\
\text { 4) Compensates for multipath propagation }\end{array}$ & $\begin{array}{l}\text { The second-order statistical information } \\
\text { of the source signal is required to be } \\
\text { exploited by the filter design }\end{array}$ \\
\hline & & $\begin{array}{l}\text { Pre-Coding/ } \\
\text { Decoding }\end{array}$ & $M \times M$ & $\begin{array}{l}\text { 1) Better than pre-nulling } \\
\text { 2) Enables advanced optimization } \\
\text { 3) Capacity optimization }\end{array}$ & $\begin{array}{l}\text { 1) Requires SI estimation } \\
\text { 2) Requires SVD of SI channel matrix }\end{array}$ \\
\hline & & $\begin{array}{l}\text { Block } \\
\text { Diagonization }\end{array}$ & $M \times M$ & $\begin{array}{l}\text { 1) Outpeforms ZF beamforming } \\
\text { 2) Precoding with adaptive power allocation to } \\
\text { optimize the sum rate }\end{array}$ & $\begin{array}{l}\text { 1) CSI is required by the base station } \\
\text { 2) SVD is required } \\
\text { 3) Power allocation satisfies KKT conditions }\end{array}$ \\
\hline & & ZF Filters & $M \times M$ & $\begin{array}{l}\text { 1) High capacity for a high SNR } \\
\text { 2) Multiple spatial streams are supported in the } \\
\text { MIMO relay }\end{array}$ & $\begin{array}{l}\text { 1) Perfomance loss in low-SNR regions } \\
\text { 2) SVD is required }\end{array}$ \\
\hline & & $\begin{array}{l}\text { Optimal } \\
\text { Eigenbeam- } \\
\text { forming }\end{array}$ & $M \times M$ & Power of the residual $\mathrm{SI}$ is minimized & $\begin{array}{l}\text { 1) Beam selection matrices are calculated } \\
\text { 2) SVD is required }\end{array}$ \\
\hline & & Maximum SIR & $M \times M$ & $\begin{array}{l}\text { 1) Improves the useful signal } \\
\text { 2) Suppresses both SI and noise }\end{array}$ & $\begin{array}{l}\text { 1) High complexity in deriving the opti- } \\
\text { mum matrices } \\
\text { 2) Channel attenuation highly impacts the } \\
\text { performace }\end{array}$ \\
\hline & & $\begin{array}{l}\text { MMSE } \\
\text { Filtering }\end{array}$ & $M \times M$ & $\begin{array}{l}\text { 1) Improves the useful signal } \\
\text { 2) Suppresses both SI and noise }\end{array}$ & High complexity \\
\hline & & TAS & $M \times M$ & $\begin{array}{l}\text { 1) Has a low complexity } \\
\text { 2) Avoids losses in low-SNR regions } \\
\text { 3) Adaptivity to varying SIRs }\end{array}$ & $\begin{array}{l}\text { 1) High-dimensional MIMO complicates } \\
\text { the best subset selection } \\
\text { 2) Unique solution for the best subset } \\
\text { selection is not always achievable }\end{array}$ \\
\hline & & 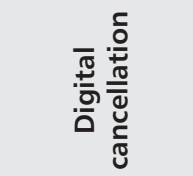 & & $\begin{array}{l}\text { 1) Residual SI after analog cancellation can be } \\
\text { eliminated in digital domain } \\
\text { 2) Modulation independence } \\
\text { 3) Addresses hidden terminal problem } \\
\text { 4) High collision-combating capability }\end{array}$ & $\begin{array}{l}\text { 1) Quantization noise cannot be reduced } \\
\text { 2) Becomes unneccessary if preceded by a } \\
\text { powerful analog cancellation } \\
\text { 3) Limited cancellation capability }\end{array}$ \\
\hline
\end{tabular}

Table 1. Performance comparison among variant SI suppression algorithms.

Combination of active and passive SI suppressions: Since none of the individual cancellation techniques is capable of satisfying the system requirements in terms of the attainable SI cancellation capability, a high-capability cancellation scheme by combining the active and passive methods is necessarily developed.
Low-complexity spatial-domain suppression approaches: Many of the existing spatial domain SI suppression methods relying on complex matrix computations may significantly erode the FD gains owing to their infeasibility. Therefore, low-complexity algorithms conceived for highdimensional MIMO channels are capable of dra- 
Theoretically, an FD system having an infinite dynamic range and perfect channel estimation can perfectly eliminate the SI signal. However, the hardware limitations, including transmit/receive signal quantization, non-linearities, inphase and quadrature mismatch, etc. all might erode the practical implementations of FD systems.

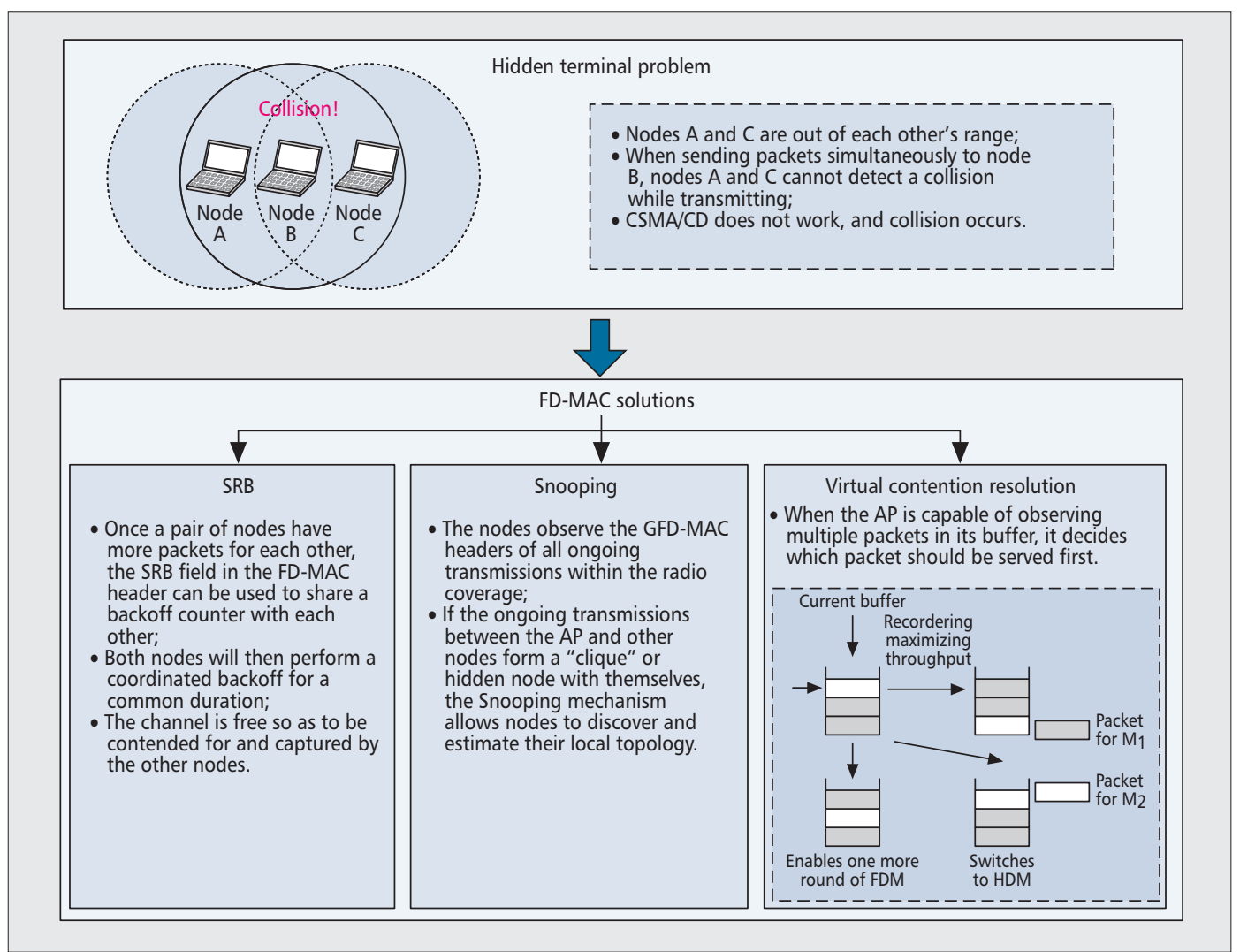

Figure 2. Mechanisms of FD-MAC protocol [4] — shared random backoff (SRB), snooping, and virtual contention resolution - can be employed for addressing the problem of the hidden terminal.

matically improving the SI cancellation capability at a reasonable hardware/software cost.

Transmit power control for improving SI suppression: A higher transmit power will definitely lead to a lower SI channel estimation error, but the absolute level of the residual SI power may still increase for a high SI power; however, the ratio between the residual error and the overall SI might be reduced.

\section{MAC Layer Protocol Design FOR FULL DUPLEX SYSTEMS}

Apart from the aforementioned physical-layer solutions, FD research opportunities have also been explored in the context of efficient MAC protocols for addressing the challenges of long end-to-end delays of network congestion and the hidden terminal problems. For instance, in [4], a new MAC protocol referred to as FD-MAC was developed and implemented for infrastructurebased WiFi-like networks to provide opportunities for all the accessed nodes while trying to maximize the overall network throughput and maintaining fairness to all users simultaneously. In order to satisfy the above-mentioned requirements, three mechanisms, shared random backoff (SRB), snooping, and virtual contention resolution, can be employed, as illustrated in Fig. 2. FD-MAC is capable of guaranteeing seamless wireless access while maximizing the FD gains. Experimental results showed that FDMAC achieves a throughput gain of up to 70 percent over its comparable HD counterpart [4].

\section{FD REALIZATIONS IN PRActical SySTEMS}

Although very few FD realizations have been implemented in commercial systems due to the technical and/or economic challenges, a substantial amount of related research has already been undertaken by addressing several challenges in this context, discussed below.

\section{HARDWARE LIMITATIONS}

In [11], the performance of co-channel FDMbased MIMO nodes was analyzed in the context of modeling their realistic hardware characteristics. Theoretically, an FD system having an infinite dynamic range and perfect channel estimation can perfectly eliminate the SI signal. However, the hardware limitations, including transmit/receive signal quantization, nonlinearities, in-phase and quadrature (I/Q) mismatch, and so on, all might erode the practical implementations of FD systems.

\section{ReCEIVER COMBINING}

Apart from the impairments imposed by SI signals and the above-mentioned hardware limitations, another challenge comes from the fact that FD-based systems might not be capable of invoking some sophisticated combining schemes such as maximum ratio combining (MRC) unless the source node and the FD-based relay are perfectly phase-synchronized. In order to address this challenge, a co-phasing scheme can be employed in the direct and relay links, facilitat- 
ing a significant coherent combining gain at the destination.

\section{HYBRID HD/FD RELAYING}

Note that FDM may not necessarily always outperform HDM in terms of throughput or channel outage probability, particularly when the FD devices suffer from high residual SI power. A hybrid HD/FD scheme, which facilitates switching between HDM and FDM, may thus be expected to outperform either HDM or FDM alone.

Scheduling for hybrid schemes: In [12], a time-domain scheduling scheme was proposed for performing a hybrid of full and half duplex relaying (FHDR) while formulating the objective function as a nonlinear programming problem. The solution of hybrid FHDR can be analytically derived by solving the above-mentioned nonlinear programming problem. Furthermore, proportional fairness in terms of all the users' end-to-end throughput can be achieved in hybrid FHDR. As compared to an equal opportunity scheduling scheme, hybrid FHDR is capable of achieving a superior performance in terms of its sum rate without sacrificing fairness among users.

Opportunistic hybrid scheme: Opportunistic duplex-mode resource allocation is motivated by resolving the fundamental trade-off between the achievable SE and the attained SI suppression capability. Explicit conditions, under which a specific duplex mode is preferred over the other, can be provided [3], enabling opportunistic hybrid FD/HD relaying to offer significant performance gains over the conventional system design that is confined to either of its constituent modes. Furthermore, the benefits of the tradeoff between the FDMs and HDMs depend heavily on the employment of transmit-power adaptation, potentially making FDMs more attractive.

Hybrid schemes in cognitive radio networks: A significant performance improvement can be attained in cognitive radio networks by developing a hybrid FDM/HDM scheme based on the classic zero-forcing criterion, provided that the multiple-antenna-based secondary transmitters have FD capabilities. The hybrid scheme has been shown in [13] to achieve almost three times the cognitive user rates provided by the HDM with the aid of the same RF chains.

\section{Full Duplex Relay Selection}

Cooperative relaying has been identified as a promising solution for effectively combating the shadowing effects to extend the radio coverage and significantly improve the channel capacity simultaneously [14]. Numerous relaying protocols, such as amplify-and-forward (AF), DF, and compress-and-forward (CF), can be employed for efficient relaying as a means of guarding against severe signal fading. Theoretically, the more relays the cooperative communication systems are equipped with, the higher the DoF provided by the relaying channels, hence promising improved performance quantified in terms of channel capacity and/or link reliability. In a multi-relay-aided cooperative communication system, activating more relays tends to attain a better DoF, because the system becomes capable of combining a higher number of independently fading signals associated with multiple relays.

However, usually orthogonal channels created in terms of carrier frequencies, time slots, or spreading codes among relays are allocated in multi-relay systems in order to mitigate the inter-relay interference, consequently eroding the increased DoF benefits due to their increased spectrum demand. In order to mitigate the above-mentioned penalty, the method of relay selection relying on channel state information (CSI) feedback has been regarded as one of the most promising solutions. The optimal relay selection scheme, in which the specific candidate relay having the "best" channel ${ }^{1}$ is activated, while deactivating the other relays, is shown to be an ideal way of optimizing the diversity order in a cost-efficient manner.

Relay selection techniques invoked in HDMbased systems have been widely studied, where the attainable benefits accrue from the fact that the system usually activates relays roaming about halfway between the source and destination. As distinguished from conventional HDM-based relay selection, in which time domain orthogonal channels must be allocated to the source $\rightarrow$ relay and relay $\rightarrow$ destination phases, FDMbased relay selection algorithms may provide a higher performance gain in terms of their outage probability and/or channel capacity due to their essential capability of concurrently transmitting and receiving in a single time/frequency slot [15]. In order to minimize the negative impact of the SI signal on the performance of FDM-based systems and optimize the signal-to-interferenceplus-noise ratio (SINR) of the source $\rightarrow$ relay $\rightarrow$ destination link simultaneously, the relay having the lowest SI power among all the candidate relays can be activated.

However, FDM-based relay-selection policies have not been widely explored, let alone evaluating their impact on the achievable system performance. Furthermore, the channel capacity of FDM-based relay selection schemes may be significantly eroded by SI power.

In this section, opportunistic DF-based relay selection schemes in underlay cognitive networks communicating over independent and identically distributed (i.i.d.) Rayleigh and Nakagami-m fading channels are considered in the context of FDM relaying. The principle of the proposed FDM relay selection scheme is described in Fig. 3.

Relay selection under Nakagami- $m$ channels: When $N=4$ and $\lambda=10$, as shown in Fig. 4 , a smaller $\bar{\gamma}_{L I}$ implies a reduction in residual SI power. The OP thus becomes a monotonically increasing function of $\bar{\gamma}_{L I}$, where $\bar{\gamma}_{L I}=0$ corresponds to perfect SI cancellation at the relay. Both passive SI suppression and active analog/digital domain SI cancellations can be invoked to reduce the residual SI power. However, for all realistic scenarios with $\bar{\gamma}_{L I}>0$, the SI cancellation would be imperfect, resulting in non-zero residual SI power in the FDM devices. Compared to FDM, HDM is capable of reducing the interference imposed on the primary users, especially when the SI level is higher. For
FDM may not necessarily always outperform HDM in terms of throughput or channel outage probability, particularly when the FD devices suffer from a

high residual SI

power. A hybrid

HD/FD scheme,

which facilitates

switching between

HDM and FDM, may thus be expected to outperform either HDM or FDM alone.

${ }^{1}$ Note that in cooperative relaying systems, the "best channel" can be defined in terms of the quality of the source $\rightarrow$ relay link, relay $\rightarrow$ destination link, or even the concatenated source $\rightarrow$ relay $\rightarrow$ destination link subject to the practical CSI feedback. 


Although FD tech-
niques are capable
of significantly
improving both the
achievable SE and
the network
throughput com-
pared to the classic
HD approach, both
efficient SI suppres-
sion and FD-based
MAC-layer protocols
are highly required.
Numerous open
challenges are still to
be tackled before
successfully imple-
menting FD devices.

instance, HDM could outperform the FDM when $\bar{\gamma}_{S R}>15 \mathrm{~dB}$.

Relay selection under Rayleigh fading channel: When $N=4, \lambda=10$, and $\bar{\gamma}_{L I}=5 \mathrm{~dB}$, we have assumed that all channel coefficients suffer from i.i.d. Nakagami- $m$ fading. The OP performance of the proposed relay selection scheme is shown in Fig. 5. Note that the Nakagami fading parameters $M=\left(m_{S P}, m_{R P}, m S R, m R D, m_{L I}\right)$ of the different cases significantly impact the attainable diversity order of the cooperative network. Furthermore, the outage performance will be more severely impacted by $m_{S R}$. As illustrated in Fig. 5, the optimum operating SNR of the proposed opportunistic relay selection scheme will always be attainable in the range of $(10 \mathrm{~dB}, 15$ $\mathrm{dB}$ ), while the attainable diversity order of underlay-based cognitive networks may be severely impacted by the fading parameter of the source $\rightarrow$ relay link.

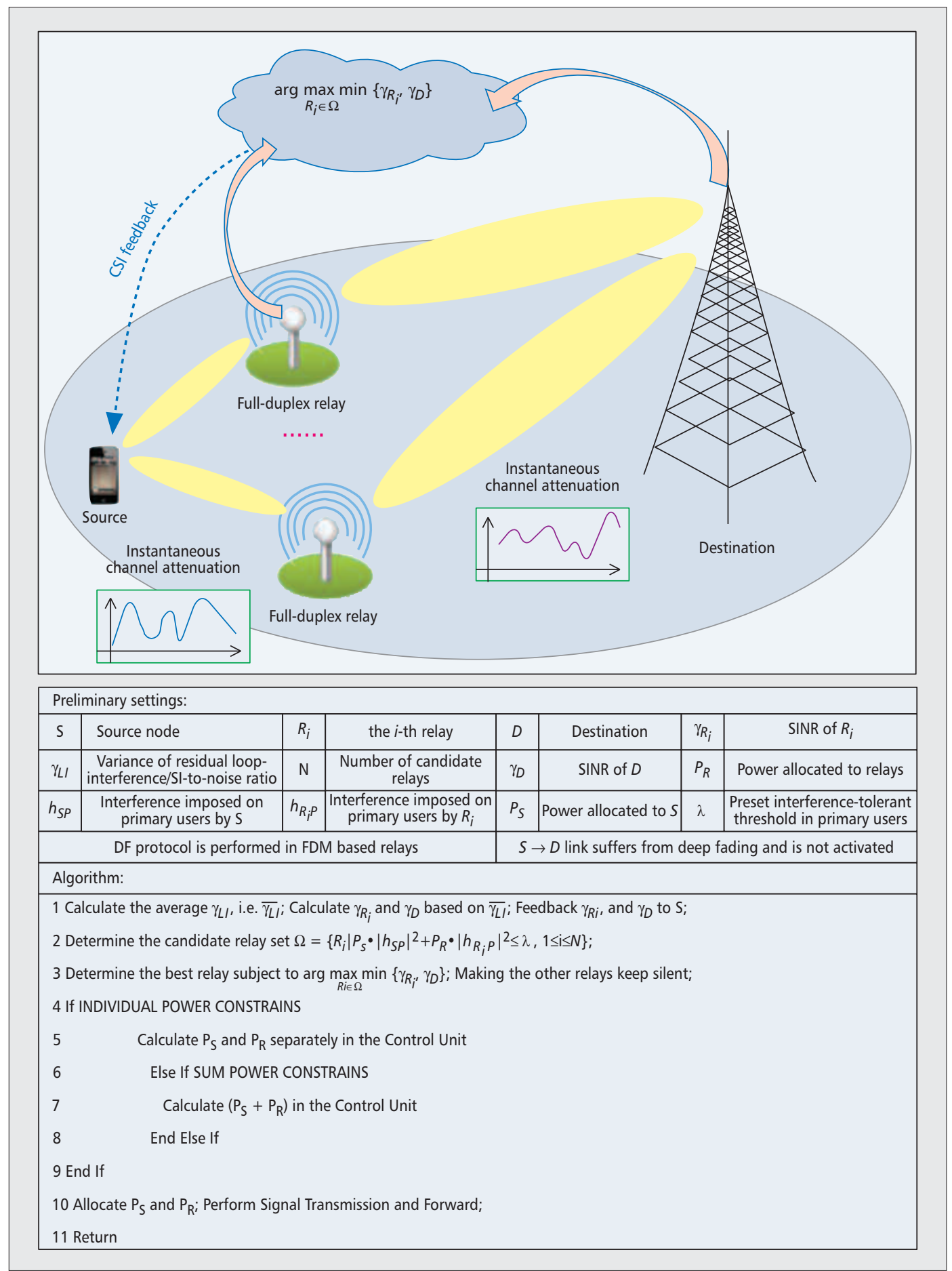

Figure 3. Opportunistic FDM-based relay selection in underlay cognitive networks communicating over i.i.d. fading channels. 


\section{REMAining ChALlENGES SND POTENTIAL FUtURE RESEARCH}

Although FD techniques are capable of significantly improving both the achievable SE and the network throughput compared to the classic HD approach, both efficient SI suppression and FDbased MAC-layer protocols are highly required. Numerous open challenges are still to be tackled before successfully implementing FD devices. In this section, general design guidelines for FD wireless communication systems are offered based on the aforementioned discussions.

\section{Remaining Challenges}

From the discussion above, some open challenges associated with FD technology have to be tackled.

FD-based device complexity issues: Carrying out powerful SI cancellation increases both the cost and complexity of FD-based devices, mainly because complex matrix computations have to be performed at the transceiver. Furthermore, the hardware limitations will also constrain the performance gain of FDM.

FD-based MAC-layer protocol design: Apart from the physical-layer solutions discussed above, a properly designed FD MAC-layer protocol, which should be backward-compatible with the existing HD-based MAC-layer protocols, is highly required for avoiding problems such as hidden terminal in multihop networks. Furthermore, the FD-based MAC-layer protocol should not unduly favor FD opportunities over HD flows, which requires the access mechanism to be capable of providing a fair opportunity for all nodes to access the shared medium.

Low energy consumption issues: Since most wireless terminals are battery-driven and have limited energy harvesting capabilities, the energy dissipation of FD-based MAC-layer protocols remains a challenging issue. It is of great importance to develop cost-efficient FD-based MAClayer protocols with low energy consumption in order to extend both the devices' battery recharge time and the network's overall survivability.

FDM in the high-SNR/data rate regime: The FDM philosophy was shown to outperform HDM in terms of capacity gain, link robustness, and/or outage probability, provided that the former operates at low to medium SNR values and information rates. Hence, expanding the benefits of FDM to the high-SNR/data rate regime is promising but challenging in practical environments.

\section{FUTURE RESEARCH}

It is worth pointing out that some of the approaches presented in this article may be further developed, as detailed below.

FDM under a wider bandwidth with a higher transmit power: The feasibility of FD technologies in systems of wider bandwidth with higher transmit power has to be further improved with the aid of improved SI cancellation capability, despite current techniques that can be effectively utilized in systems having relatively narrow bandwidth and low transmit power (e.g., IEEE

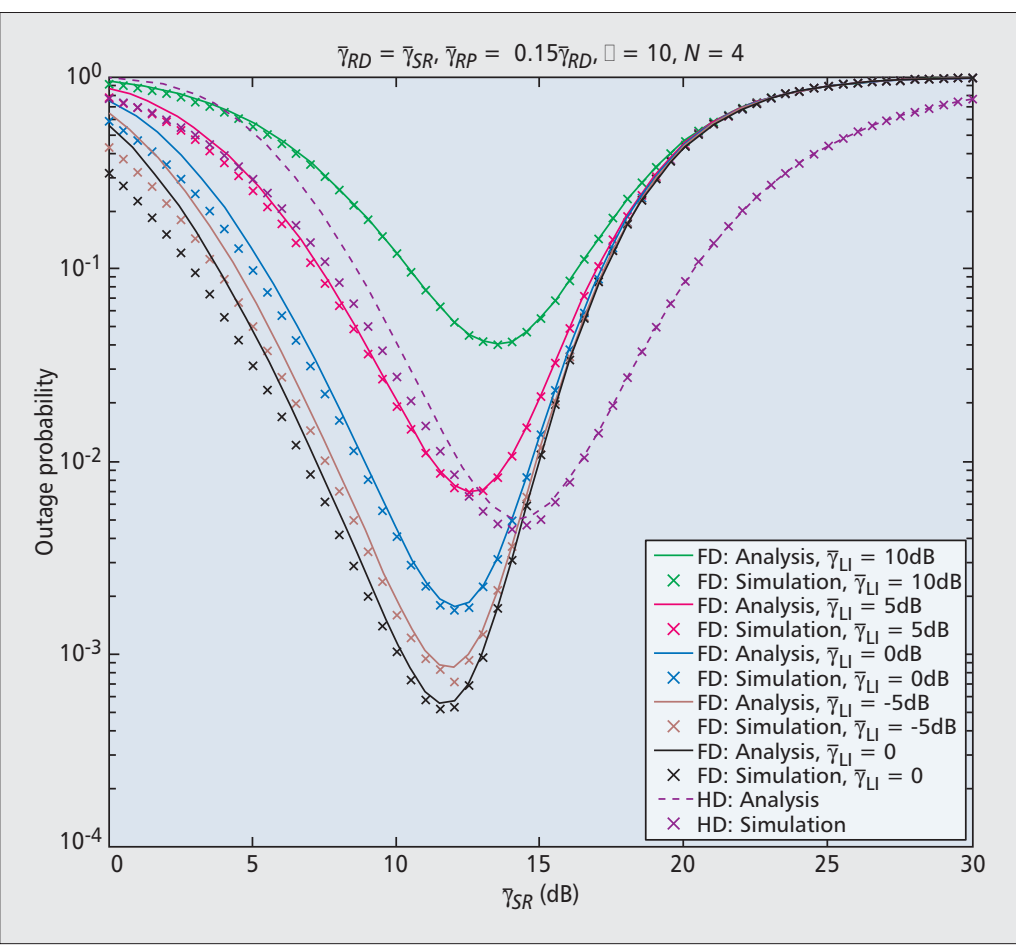

Figure 4. Outage probability vs. average SNR of the $S \rightarrow R$ links in conjunction with $\lambda=10$ and $N=4$.

\subsection{4).}

Cost-efficient spatial-domain SI suppression: Complex matrix computations are usually required in many existing spatial-domain SI suppression methods with a complexity burden that significantly hampers the realizability of FD systems. Therefore, more cost-efficient spatial domain SI suppression algorithms have to be designed specifically for MIMO channels.

FDM-based MAC-layer protocols: Many critical issues, such as the problems of hidden terminals, and multiple access collisions of distributed techniques, the requirements of low power consumption, and maintaining backward-compatibility with existing MAC protocols, cannot be readily addressed in the context of FD-based MAC-layer protocols. Thus, an appropriate MAC-layer protocol conceived for fully exploiting the FDM benefits is definitely worthy of further study.

Practical implementation of a hybrid FD/HD scheme: Although a hybrid scheme facilitating dynamic switching between HDM and FDM is capable of outperforming either of its constituent modes, the hybrid-mode-based devices have to be capable of identifying the CSI changes and promptly switching between these two modes. In the absence of a centralized controller, a sophisticated distributed approach relying on self-organization principles [16] could be employed by devices to implement a cost-efficient hybrid protocol.

Buffer size vs. PLR/delay trade-off: Since an FDM-based device has to process twice as many packets as an HDM-based device due to its essential capability of concurrently transmitting and receiving in a single time/frequency slot, both the PLR and the delay may become more 


\section{REFERENCES}

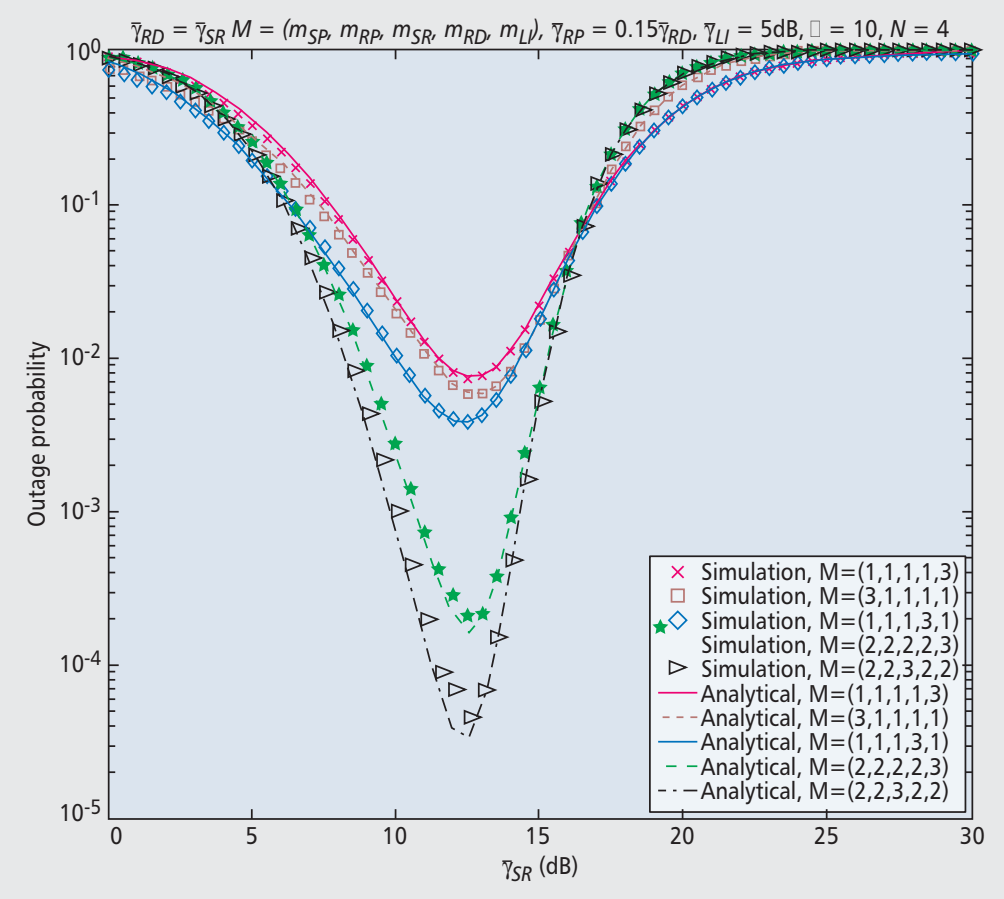

Figure 5. Outage probability vs. the average SNR of $S \rightarrow R$ links, where $\lambda=$ $10, \bar{\gamma}_{L I}=5 \mathrm{~dB}$, and $N=4$. In each simulation, the solid curves are used to stand for the analytical results, and the markers without lines denote the simulation results. severe for FDM than for HDM unless the buffer's queue length in the former is significantly increased. Nevertheless, striking the appropriate buffer size vs. PLR/delay trade-off constitutes a promising study item.

\section{CONCLUSIONS}

Since the throughput requirements cannot be readily satisfied without increasing the achievable SE expressed in bits per second per Hertz, FD technology has been proposed with the promise of nearly doubling the data rate in comparison to its HD counterpart. An FDM-based device potentially facilitates simultaneous transmission and reception within the same frequency band. One of the main challenges in implementing FD communications comes from the performance erosion induced by the SI power, which has to be suppressed/cancelled to a tolerable level. However, the family of existed SI suppression/cancellation solutions is typically based on costly hardware design and/or complex matrix computations, cost-efficient algorithms associated with low complexity are highly required for improving the realizability of practical HDM based devices. Apart from the physical-layer issues, there is also an urgent demand for highperformance low-complexity FD protocols, requiring the impact of the $\mathrm{MAC} /$ higher-layer protocols on the practical implementation of FDM-based systems to be investigated more vigorously. Last but not least, FDM-based relay selection will also play a critical role in optimizing the performance gain of multi-relay cooperative communication systems.
[1] S. Hong et al., "Applications of Self-Interference Cancellation in $5 \mathrm{G}$ and Beyond," IEEE Commun. Mag., vol. 52, no. 2, Feb. 2014, pp. 114-21.

[2] T. Riihonen, S. Werner, and R. Wichman, "Optimized Gain Control for Single-Frequency Relaying with Loop Interference," IEEE Trans. Wireless Commun., vol. 8, no. 6, June 2009, pp. 2801-06.

[3] "Hybrid Full-Duplex/Half-Duplex Relaying with Transmit Power Adaptation," IEEE Trans. Wireless Commun., vol. 10, no. 9, Sept. 2011, pp. 3074-85.

[4] A. Sahai, G. Patel, and A. Sabharwal, "Pushing the Limits of Full-Duplex: Design and Real-Time Implementation," arXiv:1107.0607, 2011.

[5] A. Sabharwal et al., "In-Band Full-Duplex Wireless: Challenges and Opportunities," arXiv:1311.0456, 2014.

[6] D. Bharadia, E. Mcmilin, and S. Katti, "Full Duplex Radios," Proc. ACM SIGCOMM, Hong Kong, 2013, pp. 375-86.

[7] X. Xie and X. Zhang, "Does Full-Duplex Double the Capacity of Wireless Networks?," Proc. IEEE INFOCOM 14, May 2014, pp. 253-61.

[8] T. Riihonen et al., "Outage Probabilities in Infrastructure-Based Single-Frequency Relay Links," IEEE WCNC, Budapest, Hungary, Apr. 2009.

[9] E. Everett et al. "Empowering Full-Duplex Wireless Communication by Exploiting Directional Diversity," Asilomar Conf. Signals, Systems and Computers, 2011, pp. 2002-06.

[10] Y.-C. Cheng et al., "Jigsaw: Solving the Puzzle of Enterprise 802.11 Analysis," Proc. ACM SIGCOMM, 2006.

[11] D. W. Bliss, T. M. Hancock, and P. Schniter, "Hardware Phenomenological Effects on Cochannel Full-Duplex MIMO Relay Performance," 2012 Conf. Record 46th ASILOMAR, Nov. 2012, pp. 34-39.

[12] M. Miyagoshi et al., "Multi-User Transmission Scheduling for A Hybrid of Full-and Half-Duplex Relaying," 2011 8th Int'l. Conf. Info., Commun. and Signal Processing, 2011, pp. 1-5.

[13] K.-K. Wong, "Maximizing the Sum-Rate and Minimizing the Sum-Power of A Broadcast 2-User 2-Input Multiple-Output Antenna System Using A Generalized Zeroforcing Approach," IEEE Trans. Wireless Commun., vol. 5, no. 12, Dec. 2006, pp. 3406-12.

[14] Z. Zhang, W. Zhang, and C. Tellambura, "Cooperative OFDM Channel Estimation in the Presence of Frequency Offsets," IEEE Trans. Vehic. Tech., vol. 58, no. 7, Sept. 2009, pp. 3447-59.

[15] H. Cui, L. Song, and B. Jiao, "Relay Selection for TwoWay Full Duplex Relay Networks with Amplify-and-Forward Protocol," IEEE Trans. Wireless Commun., vol. 13, no. 7 July 2014, pp. 3768-3876.

[16] Z. Zhang et al., "On Swarm Intelligence Inspired SelfOrganized Networking: Its Bionic Mechanisms, Designing Principles and Optimization Approaches," IEEE Commun. Surveys \& Tutorials, vol. 16, no. 1, 1st qtr. 2014, pp. 513-37.

\section{BIOGRAPHIES}

ZHONGSHAN ZHANG (zhangzs@ustb.edu.cn) PLEASE SUPPLY BIOGRAPHY

\section{XIAOMENG CHAI PLEASE SUPPLY BIOGRAPHY}

KEPING LONG (longkeping@ustb.edu.cn) PLEASE SUPPLY BIOGRAPHY

AtHANASIOS V. VASILAKOS (vasilako@ath.forthnet.gr) PLEASE SUPPLY BIOGRAPHY

LAJOS HANZO (Ih@ecs.soton.ac.uk) PLEASE SUPPLY BIOGRA$\mathrm{PHY}$ 